\title{
Nursing Cooperation in the Craniotomy of Severe Craniocerebral
}

\section{Trauma}

\author{
Daoxin $\operatorname{Jin}^{1, \text { a }}$ Yanhua Guan ${ }^{1, b}$ \\ ${ }^{1}$ Luohe Medical College, Luohe City, Henan Province, China, 462000 \\ a email: \\ b email:
}

Keywords: Severe Craniocerebral Trauma, Craniotomy; Nursing Cooperation, Routine Method, Effect

Abstract. Traumatic brain injury is a common clinical trauma, and its incidence is only inferior to that of the extremities. Most patients are admitted to hospital emergency, and the main characteristics of clinical is locality neurological deficit. The morbidity and mortality of the disease are very high, at the same time, $60 \%$ of the patients in the survivals of severe craniocerebral trauma have obvious limb dysfunction, namely limb paralysis, which seriously affects the recovery of patients' daily life ability and the quality of life. At present, craniotomy is the main treatment for severe craniocerebral trauma, and the method can delay the development of the disease, improve the clinical treatment effect and reduce the clinical mortality. However, due to the lack of ideal nursing methods in the operation of some patients, the incidence of postoperative complications is high and the prognosis is poor, causing that it is difficult to achieve the desired therapeutic effect. Related research shows that standardized process management can ensure that patients with more simplified procedures, can enhance the rescue efficiency. From January 2013 to December 2015, the author actively participated in the brain injury patient surgery treatment nursing, achieving good results, and now the results are reported as follows.

\section{Clinical Data}

From January 2013 to December 2015, there are 68 patients with traumatic brain injury receiving diagnosis and treatment in our hospital, severe traumatic brain injury in 31 cases. Operation rescued 17 cases when the author was on duty, male 13 cases, female 4 cases, age 9-55 years old, and the average age is about 34 years old. In these patients, 4 cases of epidural hematoma, 2 cases of subdural hematoma, 5 cases of brain contusion, 6 cases of complex brain injury. Typical case: patient, male, 21 years old, preoperative diagnosis: Right epidural hematoma; Left subdural hematoma; Brain contusion. Postoperative diagnosis: Right epidural hematoma; Left subdural hematoma; Skull base fracture; Brain contusion. Now the operation nursing coordination is summarized as follows.

\section{Specific Measures for Prevention and Nursing}

All patients were given routine care, indwelling catheter, on this basis, implementing nursing 
cooperation: (1) preoperative coordination. Ensuring airway patency. For patients with severe traumatic brain injury, because of the sudden increase in intracranial pressure, they are likely to be accompanied by vomiting, increasing the incidence of aspiration. Therefore, the patient should be allowed to the side of the head, timely removal of removable partial denture, helping patients to clear the nose and mouth pharynx clot and vomit. For patients with impaired consciousness, swallowing and cough reflex, the phlegm blocking is easy to cause suffocation, so it need to suck out the secretions in the patient's respiratory tract. Open venous access. After entering the operation room, nurses should help patients to establish at least 2 venous channels, for patients with poor circulation and vascular collapse, it should conduct central venous catheterization to ensure the smooth completion of the infusion, blood transfusion and central venous pressure monitoring. Medication nursing. Following the doctor's advice before surgery, by intravenous antibiotics to prevent infection, giving the patient water treatment, hormone and other methods of treatment to reduce the intracranial pressure; Prevention and nursing of falling bed. For coma patients with restless, the patient should be fixed on the operating table with the constraint belt to 0 avoid falling bed. Body position nursing. Before operation, the nurse should instruct the patient to put the body position. Body position care should be based on patient comfort, close observation of the patient's vital signs, to maintain a smooth breathing, which is conducive to the operation of the rescue. (2) Intraoperative coordination. Observation of vital signs: During the operation, nurses should strengthen the monitoring of ECG, observe the changes of pulse, respiration, blood pressure and blood oxygen saturation, accurately record the urine volume, urine color, observe the changes of body temperature and peripheral circulation in patients with close observation, do a good job in patients with insulation measures. Maintain airway patency: Nurses should ensure there is no accumulation of carbon dioxide in the body of the patient to prevent the increase of intracranial pressure. Oxygen is given in the operation, dynamically monitoring the blood oxygen partial pressure, carbon dioxide partial pressure, blood oxygen saturation and respiration, adjusting oxygen flow rate in time according to the patient's condition. The coordination during the operation: In the craniotomy, the different steps must be strengthened to reduce the amount of bleeding. Before opening the dura, the hemostatic items, including: gelatin sponge, hemostatic cotton, cotton piece, etc., must be fully prepared; Before closing the head, the suture, needle, artificial dura mater and connecting piece must be prepared, and the nurse must be skilled, rapid to reduce the amount of bleeding during perioperative period. Medication during operation: In the period of perioperative, the drug used by patients is relatively more, before opening the dura, it should reduce intracranial pressure and avoid intraoperative tissue prolapse; For patients with acute brain injury, most need to use dehydration agent before the operation, because a lot of blood loss can easily lead to insufficient blood volume, causing hemorrhagic shock. Therefore, the operation should help patients with blood transfusion, infusion, for the conditional patients, it can use the heater to heat the liquid and blood to 25-35 centigrade, closely observing the vital signs, timely helping patients to correct shock and so on, to avoid the brain edema. Sterile operation: In the period of perioperative, it must strictly follow the aseptic operation, conducting intravenous infusion of antibiotics according to the patient's operation time, at the same time, the operation should be kept clean, sterile, and the surgical instruments should be placed in an orderly to reduce the transmission of equipment. Postoperative nursing care: After surgery, it should closely observe the patient's vital signs, understand the patient's recovery, monitor the patient's heart rate, blood oxygen and blood pressure. Intracranial hemorrhage is easy to occur in $24 \mathrm{~h}$ after the operation, reach its peak within $48-72 \mathrm{~h}$, therefore, postoperative monitoring within 3days of intracranial pressure is very important. In order to reduce the brain stem displacement to endanger life, it needs to keep the patient's head side to the healthy 
part, with head elevation 15 degrees -30 degrees, maintaining 24 - 48h.

\section{Results}

Through the implementation of nursing care for patients with severe traumatic brain injury, the patient achieved the desired results, and the surgical procedures reduced the incidence of hemorrhagic shock, infection and other complications, improved the success of patients with surgery, getting the recognition of the patients and their families. The 68 patients were aware of the disease pathogenesis, treatment methods and the treatment process, and they were able to know the relevant notes. At the same time, strengthening patients with drug care, aseptic manipulation during operation, maintaining airway patency and the observation of vital signs can ensure the smooth completion of the surgical treatment of patients, making patients and their families to have a comprehensive understanding of the surgical program. All patients actively cooperated with the nursing staff, getting a significant nursing effect. After surgery, the nurse should take the initiative to communicate with patients, strengthen psychological counseling to help patients to establish the confidence to overcome the disease, and according to the patient's recovery, the nurse should help the patient to do the corresponding functional exercise to promote the early recovery of patients.

\section{Conclusion}

Severe brain injury is a common disease in clinic, and the disease has the characteristics of danger, serious, urgent, risk and so on. In the early stage of disease, if there is a lack of positive and effective treatment, other diseases will be induced, and the severe cases will threaten the life of patients. As we all know, due to high central brain lesions, the severe head injury patients lost the control of the low position, which is easily lead to further loss of balance movement function, thereby affecting the balance of the body and exercise capacity.

At present, the clinical treatment for patients with severe brain injury is mainly based on surgical treatment, and this method can improve the patient's symptoms, improve the clinical treatment effect and avoid the further development of the disease. However, due to the lack of ideal nursing methods, the incidence of complications is high during the operation, causing it is difficult to achieve the desired therapeutic effect, and affecting the health of patients. Therefore, the operation process not only requires the nurse to have a good psychological quality, a high degree of professional responsibility, a solid professional knowledge and professional skills, but also requires the nurse to have a wealth of clinical experience and keen observation ability. In summary, the implementation of nursing care for patients with severe traumatic brain injury achieved the desired results, and this method can guarantee the smooth completion and improve the rescue efficiency of the operation, so it is worthy of popularization and application.

\section{References}

[1] Li Kaiyu, Li Bin, Chen Changchun. Analysis of standard large trauma craniotomy in the treatment of severe craniocerebral injury [J]. Modern medicine, 2012,36:94-96.

[2] Dong Wenming, Zhang Zhixue, Dong Yuping. Acute encephalocele in severe craniocerebral trauma craniotomy in 26 patients [J]. Modern preventive medicine, 2010,24:4685-4686.

[3] Zhao Bi, Lu Xianzhen, Su Song, Zhu Yunfei. Clinical analysis of standard large trauma craniotomy in the treatment of 48 cases of severe traumatic brain injury [J]. Chinese Journal of Clinical Department of Neurosurgery, 2008,10:621-622.

[4] Zheng Buyi. The value of intracranial pressure monitoring in patients with severe traumatic 
brain injury after craniotomy [A]. Department of Neurosurgery, Zhejiang Provincial Medical Association. Department of Neurosurgery Academic Exchange Conference[C]. Department of Neurosurgery, Zhejiang Provincial Medical Association: 2015:2.

[5] Het Shougang. Clinical analysis of acute encephalocele in severe craniocerebral trauma craniotomy[J]. Chinese community doctor, 2015,30:24-25. 